\section{Pevent}

MRC Experimental Parasitology Group University of Nottingham Nottingham NG7 2RD UK.

\title{
Do Hookworms Elicit Protective Immunity in Man?
}

\author{
J.M. Behnke
}

The two main species of human hookworm, Ancylostoma duodenale and Necator americanus (Table 1), are together believed to infect about 900 million people - mainly in tropical countries where adequate sanitary facilities may be lacking. But interactions between the two species, and their relative contributions to observed age-related infection patterns and seasonal cycles of transmission, continue to engender controversy. People tend to remain susceptible to infection throughout life, even with constant exposure to the infective stages. So what role does human immunity or resistance play in the epidemiology and control of infection?

In this article, Ferzy Behnke reviews the epidemiology of hookworm infection in the light of current understanding of mechanisms involved in host responses to infection and hookworm evasion of those responses. As he stresses, much further work is required.

It is often stated that $A$. duodenale is a northern species - the principal agent of human hookworm disease north of $20^{\circ}$ latitude ${ }^{1,2}$. In contrast, $N$. americanus is considered a southern species. However, this distinction is not so simple because foci of $A$. duodenale have been found in many parts of Africa ${ }^{3-6}$. Eggs of the two species are not easily distinguishable $^{1}$, so estimates of the relative intensity of infection in mixed infections depend either on the recovery of adult worms from the faeces after chemotherapy or on culture of eggs to the infective stage, when the larvae can be individually identified. Both are labour intensive exercises and it is not surprising that many workers have not confirmed the identity of the parasites beyond their recognition as hookworms ${ }^{1}$. This is a continuing problem in hookworm research, but nevertheless it is quite clear that there are important differences in the biology and host-parasite relationships of $N$. americanus and $A$. duodenale. These differences may account

Table I. The most commonly studied species of hookworm

\section{Necator americanus}

Age-intensity profiles of hookworm infection usually show a peak or plateau of infection in the 20-30 age group which rarely falls in older age groups. This does not support the idea of an acquired immunity to infection that could cause a decline in infection intensity omongst older groups.

However, longitudinal studies report fluctuations in apparent worm load that suggest regular loss and reacquisition of parasites.
Various other species of Necator and Ancylostoma are known from taxonomic studies on animal parasites but none of these has been studied in the laboratory. for some of the inconsistencies in epidemiological findings from different parts of the World ${ }^{7}$, and there is an urgent need to define accurately the extent of infection with both organisms in future studies.

\section{Longevity and Egg Output}

Hookworms are considered to be longlived parasites. Few longitudinal studies have been carried out but age-intensity profiles of affected communities reveal that children tend to acquire infection within the first 4 years of life (Fig. 1). This is followed by increasing intensity of infection (monitored through faecal egg counts and expressed as the mean number of eggs per gram of faeces-EPG) which peaks or plateaus in the 20-30 age group. Thereafter EPGs tend to remain stable into late life or even rise further in old age ${ }^{8-10}$. One study in Nigeria reported a decline in mean EPG in the 45+ age group to a level comparable to that of $1-4$ year old children ${ }^{11}$. The identity of the hookworm was not given but other studies in Nigeria report that $N$. americanus accounts for around $88 \%$ of the hookworm infections. No other survey to date has yielded quite comparable results, and it is more common for the age-intensity curve to stabilize in adult life - particularly in regions where only Necator occurs, such as Gambia ${ }^{9}$ and Puerto Rico ${ }^{12}$. A study in Taiwan, where $A$. duodenale is dominant, found that the mean EPG fell in females aged $45+$ but continued to rise in men among whom the heaviest infections were in 55-60 year age group ${ }^{8}$. Similar observations have also been made in India ${ }^{10,13}$.

Collectively these studies do not lend support for the existence of immunity to hookworms under field conditions. However, longitudinal studies across 1-2 transmission seasons report fluctuations in EPG 


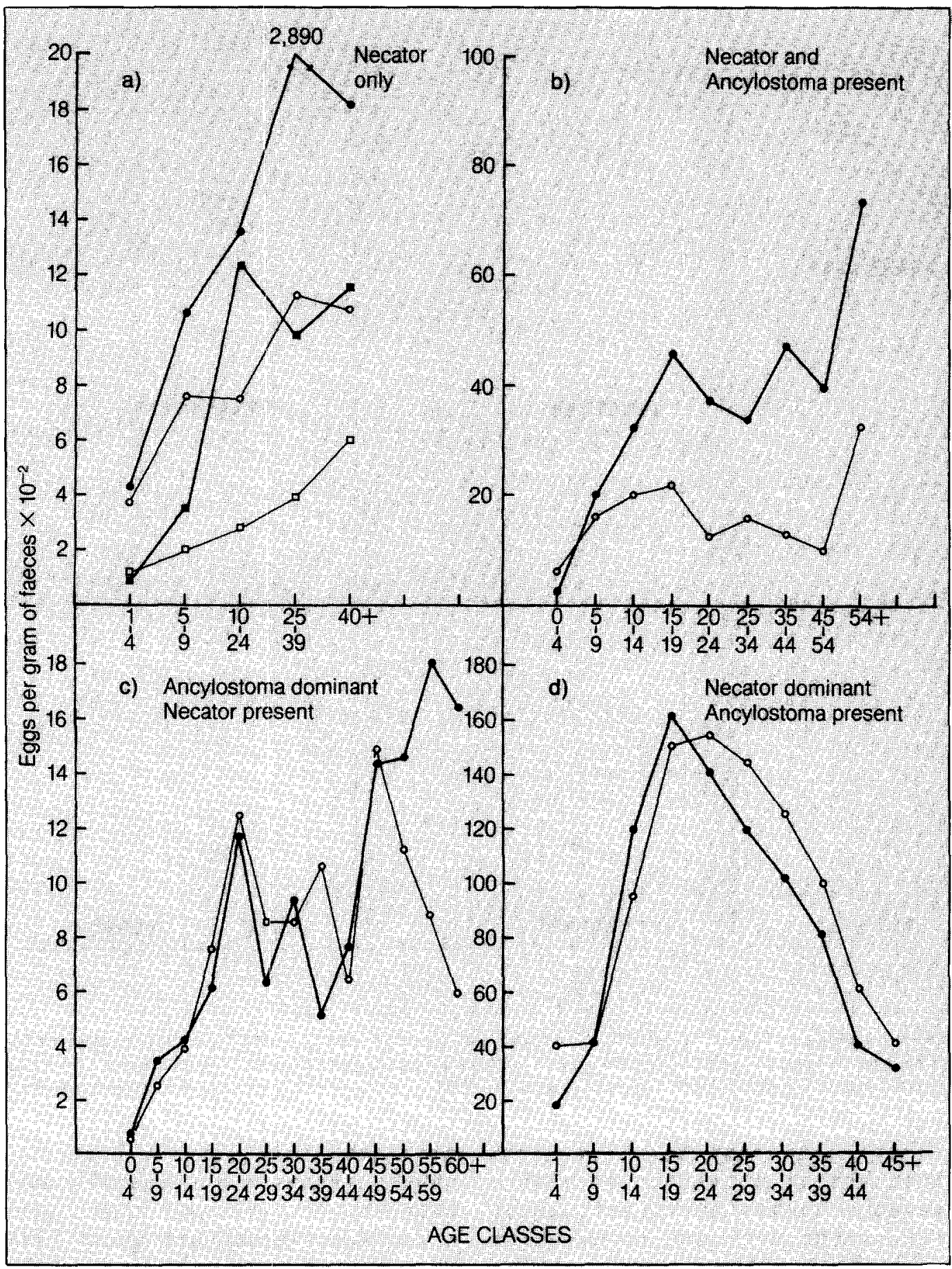

which suggest that parasites are regularly lost and subsequently reacquired in the following transmission season. It is exceptional for hookworm larvae to be continuously available throughout the entire year, but even then the density of infective larvae in contaminated soil oscillates with the seasons. In Nigeria ${ }^{5,14}$ the density of hookworm larvae increased over 10-fold from very few in the dry months (January-February) to a maximum in July-October when rainfall was frequent. In Tanzania ${ }^{3}$ and West Bengal ${ }^{13}$ transmission seasons are sharply delimited by the hot dry climate between monsoons.

Early studies in India ${ }^{15}$ first suggested that faecal egg counts rose during the early part of the rainy season, peaked, and declined in the dry season (Fig. 2). This feature of hookworm epidemiology has been recently confirmed ${ }^{16}$ and attributed to population changes in $A$. duodenale rather than $N$. americanus. In this work ${ }^{16}$, the population studied was about equally affected by both species, and the authors concluded that $A$. duodenale was a relatively short-lived species whilst $N$. americanus gave rise to more stable, long-lasting infections.

This conclusion is supported by several other studies which reveal that $N$. americanus infections are more stable in the field under endemic conditions. Longitudinal surveys in Brazil ${ }^{17}$, Gambia ${ }^{9}$ and East
Fig. I. Age-intensity curves for hookworm infection in four different regions (open points - females, closed points - males) $a-$ Gambia9 (circles Marakissa community, squares-Mandinari community). $b$ - West Bengal, India ${ }^{45}$. c-Taiwan ${ }^{8}$. d-Nigeria"l.

Hookworms are long-lived parasites. Necator americanus can survive in the gut for up to 15 years, while Ancylostoma duodenale lives for more than 12 months-probably up to 5 yeors. 
c)

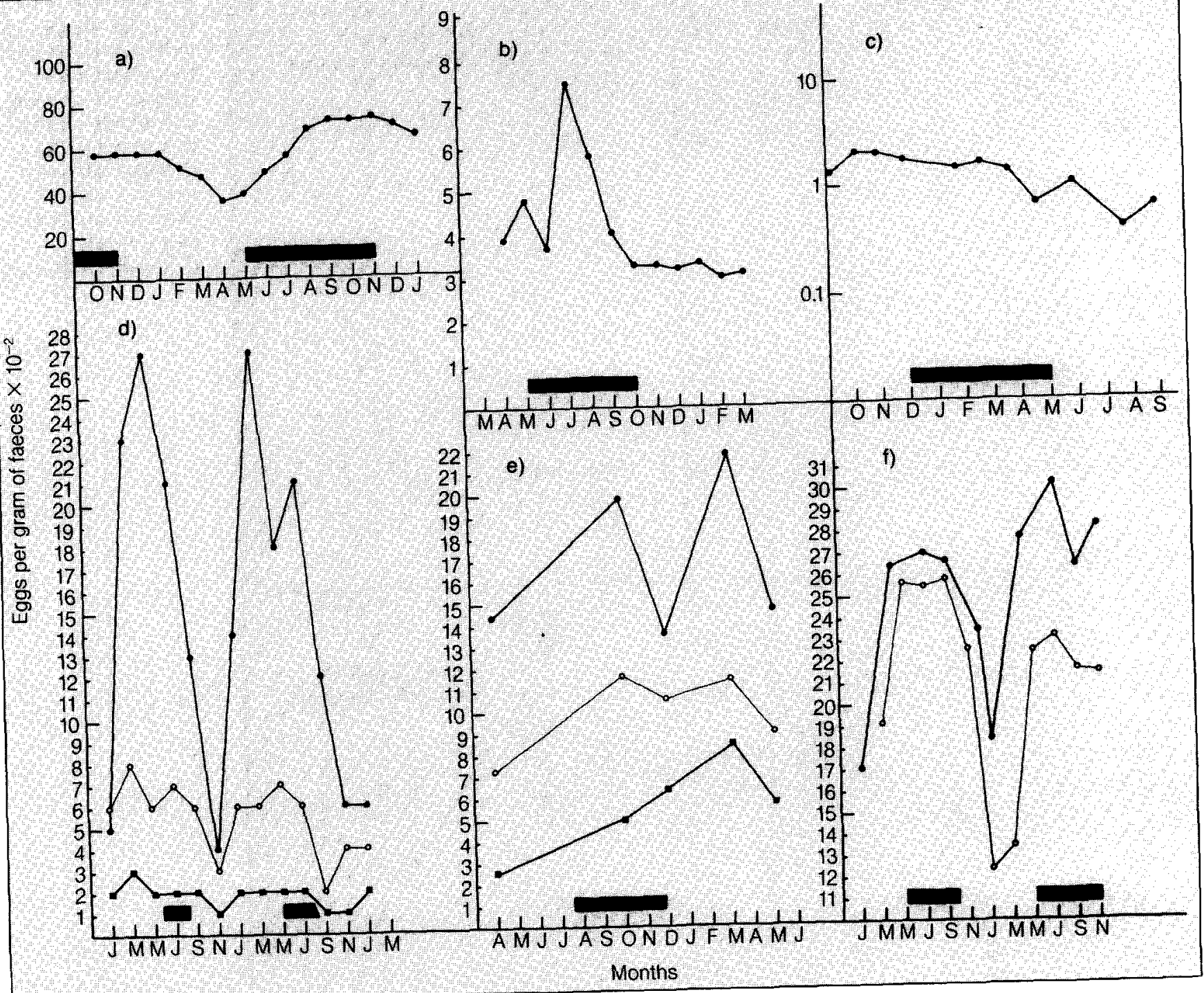

ig. 2. Longitudinal studies of hookworm infection. (a) Nigeriall; (b) India 15 ; (c) Tanzania ${ }^{3}$; (d) Taiwan ${ }^{8}$ [ - group in which the highest egg count reached at least 3000; $0_{-}$group in which the highest egg count was 1000-2999; - group in which the highest egg count was 400-999]; (e) Gambia [ [ - males,

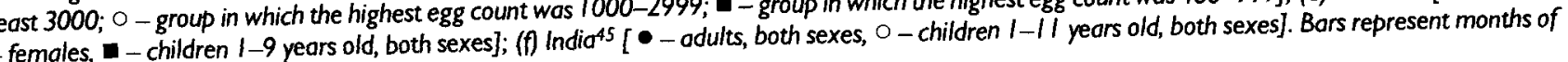
heovy rainfall in each study.

Africa ${ }^{4}$ all suggest that where $N$. americanus is the dominant species, there is no obvious pattern of regular worm loss and reacquisition, despite short term fluctuations in EPG. The exception is amongst children still acquiring worms, where an upward trend is usually discernible in the transmission season ${ }^{9}$. Further evidence for the stability of $N$. americanus infections comes from monitoring individuals who have been isolated by hospitalization or imprisonment or have been self-infected. The worms can survive for up to 15 years ${ }^{18}$ and loss is gradual over this period. Nevertheless there is still some disagreement. Nwosu and Anya ${ }^{5}$ found seasonal oscillations in EPG coinciding with rainfall, but their data were based on a population in which there was $88 \%$ prevalence with $N$. americanus. At the low point in the dry season, EPGs were still quite high (4000) and the fluctuation in egg counts above this baseline may therefore represent loss and gain of $A$. duodenale, which although in a minority in this community, is known to be considerably more fecund than $N$. americanus ${ }^{16}$. In areas where A. duodenale is the dominant species, EPGs fluctuate in regular annual cycles in relation to rainfall and transmission season ${ }^{8,16}$.

The interpretation of annual cycles in EPG is not straightforward. It is widely assumed that egg counts reflect the number of parasites harboured by the host, but this is not a reliable indicator of parasite burden ${ }^{19}$. Infections of only male worms would not be recognized through faecal egg counts, and larval burdens would also be missed. Furthermore there is evidence for a density-dependent reduction in female fecundity, and the possibility that female worms reduce or even cease egg output in the dry season when transmission is virtually abolished. The relationship between EPG and worm burdens (differential counts for both species) urgently requires examination, and a comparison of worm burdens 
during dry season and transmission season, following chemotherapy, would go a long way to providing relevant answers.

\section{Arrested development}

Another factor which may contribute to fluctuating egg counts is arrested development (hypobiosis) in $A$. duodenale. Individuals infected with the West Bengal strain took 22-38 weeks to develop mature worms - considerably longer than the normal prepatent period ${ }^{20,21}$. These subjects were not exposed to further infection and it is conceivable that the parasites had lain dormant in the intervening period. Reinterpretation of Maplestone's earlier work ${ }^{22}$ reinforces the concept that in West Bengal, $A$. duodenale may have the capacity for arrested development. Egg counts on prisoners who commenced their sentences just after the monsoon season showed that EPGs fell steadily until February, but from March onwards increasing quantities of eggs were passed, peaking in May despite the absence of reinfection. Field observations provide further support ${ }^{20}$. The increase in egg production usually associated with the monsoon season actually began to be apparent in West Bengal in late April when ground conditions were still inappropriate for transmission. A temporary reduction in the fecundity of female worms could explain these observations, but it seems more likely that arrested larvae from the previous season began to mature in advance of the new transmission season, synchronizing their reproduction to environrnental conditions most likely to ensure further infections. $A$. duodenale in the field may therefore have a relatively short survival time, adult worms declining in the dry season leaving newly acquired larvae to survive the months least suitable for transmission in an arrested state. In contrast, $N$. americanus continue to produce eggs steadily throughout the year and so, in regions where both species coexist, faecal cultures in the dry season yield a greater proportion of $N$. americanus larvae ${ }^{16}$.

\section{Worm Loss and Immunity}

Does the regular loss of adult hookworms imply immune mediated expulsion of worms and acquired immunity? Evidence for the induction of protective immune responses by hookworms in man is still scarce, although recent work supports the idea that some people are predisposed to reinfection following treatment with anthelmintics 23,24 . It may be that $A$. duodenale burdens decline each year through natural senility, but such a short life span is not compatible with Kendrick's study on experimentally infected prisoners in Madras penitentiary ${ }^{25}$. All the prisoners monitored for 12 months or more remained infected, and in some, egg output was maintained for over 5 years. A. duodenale therefore can live for longer than 12 months under conditions free from reinfection. In endemic regions the life span of adult worms is shorter - Nawalinski et al. ${ }^{16} \mathrm{cal}$ culated a $60 \%$ annual turnover for $A$. duodenale in West Bengal.

These observations imply that although a significant proportion of adult $A$. duodenale are lost in the post monsoon season, some would survive together with arrested larvae (and $N$. americanus) to continue the cycle into the next season. Perhaps worm loss is selective - acting on individual worms rather than on the entire worm burden. Possibly there are highly localized responses to individual parasites in the intestine, or alternatively age-related changes within the parasites may increase their susceptibility to host effector mechanisms.

It is likely that humans express a range of response levels to hookworm infection, as do sheep to infection with Haemonchus contortus ${ }^{30}$, and inbred mouse strains to intestinal nematodes ${ }^{26-29}$. Studies in Tanzania for example, where $A$. duodenale and $N$. americanus coexist in almost equal proportions, show that in some children, egg production was rapidly curtailed after the transmission season - while in others, more stable EPG patterns emerged with varying degrees of fluctuation. Individuals who do not pass hookworm eggs have been recognized in all studies despite the generally high prevalence in affected communities. But it is extremely difficult to distinguish between variation in exposure to hookworm larvae, and genetically determined capacity to respond immunologically to infection. Carefully combined epidemiological and immunological observations, continued over several seasons on populations showing contrasting hookworm burdens, single and mixed species infections, would provide much needed information.

\section{Predisposition to Infection}

Volunteer studies with $N$. americanus show no evidence of acquired resistance to reinfection in terms of reduced parasite establishment, delayed maturation or shorter patent period ${ }^{31}$. Field studies in which populations were treated with anthelmintic suggest that reinfection soon follows after chemotherapy but precontrol levels in the intensity of infection are reached only after several years (Fig. 3). Schad and Ander-
People express a range of response levels to hookworm infection. Individuals who do not pass hookworm eggs have been recognized in all studies, despite the generally high prevalence in affected communities. Moreover, there is good evidence from studies of chemotherapy followed by natural reinfection, that certain individuals are predisposed to heavier infections than others. But it is very difficult to distinguish between predisposition due to particular levels of exposure to infection, and predisposition through a genetically determined capacity for a particular level of immunological response. 

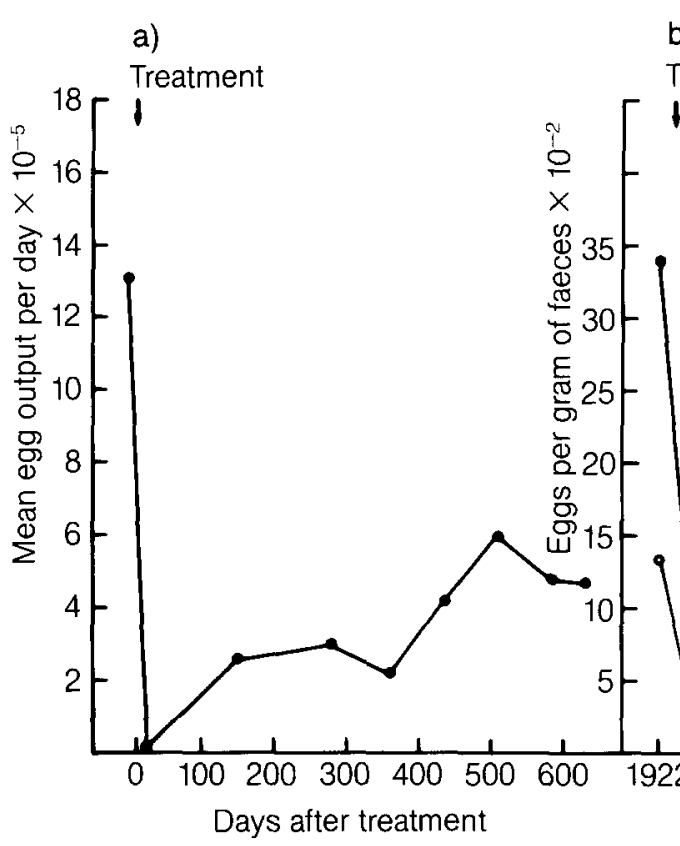

b)

Treatment

1

(1) c)

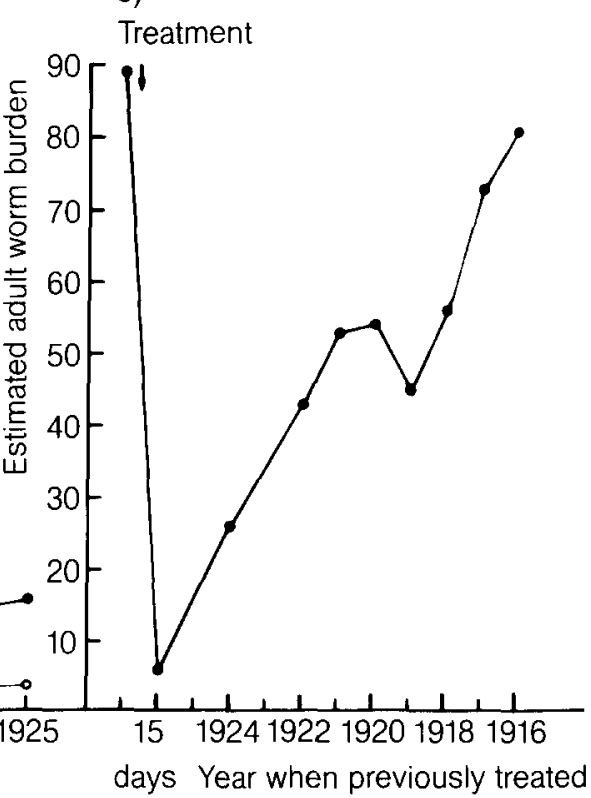

Fig. 3. Reinfection with hookworms after treatment with anthelmintic. (a) India ${ }^{23}$ [all ages and both sexes]; (b) Puerto Rico ${ }^{32}$ [ $\bullet-$ males, $0-$ females]. Both figures show mean faecal egg counts before and after treatment. The arrow indicates the point at which anthelmintic was administered. (c) Ceylon ${ }^{33}$, showing the number of worms estimated from foecal egg counts to have been harboured by tea plantation labourers and village residents in north and central Ceylon. Individuals were grouped according to the year in which they had previously been treated for hookworms. The first point shows the mean worm recovery from 3825 persons who had never before received treatment. The second point is based on 1728 persons re-examined within 15 days of treatment, and the remaining points refer to groups of 154-635 individuals who were previously given anthelmintic in the year indicated. The data for 19/6 are based on only 29 persons. (A group of 18 individuals previously treated in 1923 had a mean worm burden of 101 . This point is not shown.)

son $^{23}$ found that only $30 \%$ of the precontrol level was restored 630 days after chemotherapy - in agreement with earlier work ${ }^{32,33}$. This slow reacquisition of adult hookworms contrasts with the relatively more rapid reinfection rates observed after elimination of Ascaris lumbricoides, where pretreatment intensity has been reported within 12 months of chemotherapy ${ }^{34}$.

Two recent studies 23,24 found significant correlation between worm burdens before, and many months after, treatment. Individuals heavily infected before treatment, subsequently reacquired above average worm burdens - supporting the idea that they are predisposed to heavy infections. But although it is tempting to suggest that predisposition may be linked to genetically determined susceptibility and resistance to infection, this hypothesis has not yet been specifically addressed in appropriately designed epidemiological studies.

\section{Antibody Responses to Hookworms}

Antibody responses to hookworms have been documented by a variety of assays using L3 and adult worm homogenates ${ }^{31}$. Naturally infected patients and volunteers infected with $N$. americanus show elevated haemagglutinating antibody, complement fixing antibody, raised $\mathrm{IgE}$ and anti-parasite acetylcholinesterase responses. Yet they remain susceptible to infection ${ }^{31}$. There is no evidence for resistance to human hook- worms involving systemically circulating antibody.

Among this array of responses there may be host protective responses which are masked by non-protective responses to the numerous antigens in the parasite preparations. Local intestinal responses have generally been ignored but there is some evidence that IgA may figure prominently in the host-parasite relationship. Kumar et al. 35 reported that IgA in the intestinal aspirates of hookworm patients was depressed, but normal levels were restored after chemotherapy. It may be that the presence of hookworms led to altered IgA secretion or reduced IgA half-life in the gut lumen, or the depressed IgA may reflect inactivation of this immunoglobulin by worm products. Hookworms are known to secrete proteases which are believed to aid in digesting the bolus of host tissues taken in during feeding 31,36 . Some of these products may be directed at IgA, as occurs among other microorganisms living on mucosal surfaces ${ }^{37}$.

Experiments with $A$. ceylanicum in dogs lend support for the importance of $\operatorname{IgA}$ in hookworm infection ${ }^{31,38}$. A vigorous parasite-specific IgA response was detected in dogs which resisted a challenge infection administered 28 weeks after primary exposure to infective larvae. In contrast, no such response was evident in dogs vaccinated with adult worm antigen, even though they 
were equally resistant to challenge. Specific IgG levels however, were considerably higher in both groups and this may have reflected responses to the somatic migratory stages: the IgA response detected in reinfected dogs may have been the consequence of enhanced secondary responses in the intestine. Vaccinated animals which did not show an elevated IgA response to challenge would not have experienced local mucosal stimulation during vaccination and therefore would not be primed for an anamnestic response to challenge.

\section{Evasion of Immunity}

If IgA is involved in protective immunity to hookworms, it appears that the parasites have evolved a counter-strategy to inactivate this immunoglobulin class in the gut. Hookworms may also secrete immunomodulatory components to inactivate local immune responses. Other species causing chronic infections are known to do this ${ }^{39}$, but there is yet no evidence that comparable strategies have been evolved by hookworms ${ }^{31}$. Antigens presented orally can induce life long tolerance of cell-mediated responses to dermal challenge and longlasting tolerance of antibody responses, whilst initiating local immunity in the form of specific $\operatorname{IgA}{ }^{40}$. Hookworms reside in the gut lumen but their antigenic presentation is not directly akin to the oral route. Hookworm mouthparts penetrate deep into the gut mucosa and their excretory products probably enter the circulation - bypassing the normal mucosal-Peyer's patch route of antigen entry from the intestine. The possibility that hookworms interfere with the immunoregulatory circuits in the intestine has not yet been examined experimentally but the hamster models use of hamsteradapted strains of $N$. americanus and $A$. ceylanicum ${ }^{31,44}$ now offers the possibility of exploring these aspects of the hookworm host-parasite relationship.

Observations on the feeding activity of the dog hookworm, A. caninum, have shown that parasite attachment to the gut mucosa is temporary, lasting for $4-6$ hours at $\operatorname{moss}^{41}$. The lesions created during feeding are rapidly repaired by the host but areas of infiltration by inflammatory cells have been observed around parasites embedded in host mucosa ${ }^{31}$. The parasites regularly change their feeding sites and may thus avoid potential damage which could be inflicted by inflammatory cells. It may be that the ability of worms to relocate is diminished with age so that, for example, older $A$. duodenale may be more readily caught in lesions around feeding sites than younger worms or $N$. americanus.

There is some evidence that hookworms have evolved strategies for evading antibody responses. Adult $A$. caninum shed immunoglobulin from their cuticle, whilst dead parasites bind antibody which can be detected using immunofluorescent techniques ${ }^{42}$. Studies with $N$. americanus surface-labelled with ${ }^{125}$ I suggest that they also shed surface antigens ${ }^{31}$. This may be an evasive mechanism preventing antibody from adhering to the parasite, although it is difficult to envisage how antibody bound to cuticle could affect hookworm viability.

A specific and continuous intestinal antibody response may explain epidemiological and experimental observations for the slow loss of hookworms. Such a response may cause the gradual deterioration of older worms but not affect young parasites freshly recruited from the environment which still have potent evasive mechanisms. It would also explain why $A$. duodenale can be lost in the post monsoon season leaving $N$. americanus unscathed. A separate response against the latter species would be required, and this may be less efficient because the parasite is more robust or employs more effective evasive strategies than $A$. duodenale.

\section{Strategies for Vaccination}

Whatever the nature of immune involvement in hookworm infection, it is clear that the parasites have the upper hand in most people living in endemic areas. A vaccine against hookworms must therefore be a considerable improvement on natural human responses to these parasites. However, it is quite possible that antigens will be identified which are not normally made available to the host during natural infection. Two such possibilities are apparent - high affinity antibodies against key components of the various nematode sensory receptors ${ }^{43}$ may block sensory input and so inhibit parasite attachments, and, because hookworms pass large quantities of serum through their intestines, a serum antibody response against the parasite intestine may cause sufficient damage for the worms to be lost. During natural infection, such antigens may never be exposed or may present insufficient antigenic challenge to the host, but their isolation may be feasible using fresh parasites grown in hamsters enabling vaccine production through gene cloning and antigen synthesis by recombinant DNA methods ${ }^{46}$.

There is considerable scope for future
A specific and continuous intestinal antibody response may explain epidemiological and experimental observations for the slow loss of hookworms. Such a response may cause the gradual deterioration of older worms but not affect young parasites freshly recruited from the environment which still have potent evasive mechanisms. It would also explain why A. duodenale can be lost in the post monsoon season leaving $N$ americanus unscathed.

Patients infected with hookworms display a wide range of immunological responses. Among them. there may be protective responses which are masked by non-protective ones. But potential strategies for vaccination might make use of antigens which are not normally made available to the host during natural infection for example with antibodies directed against the nematode sensary system, or serum antibodies against the nematode intestine. 
Nextmonth in Parasitol. og. Todoy. Peter Hotez Nuyen Le Trang and Anthony Cerami revew progress in characteriz. ing hookwom antigens: and discuss the potertial for improved immuno. dignosis and possible vaccination

\section{PARASITE Pathology}

Medical Research Council Laboratories,

Fajara, Banjul,

The Gambia research on hookworms, using recently developed animal models 44 and by epidemiological projects linking immunological assays with resistance and worm loss in the field. Understanding the mechanisms involved in worm loss will be an important step in potential vaccine production, and I hope that a concerted effort to apply the tools of modern experimental medicine will help to elucidate aspects of hookworm biology which have eluded experimenters in the past.

\section{References}

1 Banwell, J.G. and Schad, G.A (1978) Clin. Gastroenterol. 7, 129-156

2 Foster, W.D. (1965) A History of Parasitology, E \& S Livingstone Ltd, Edinburgh and London

3 Sturrock, R.F. (1967) E. Afr. Med. F. 44, 142-149

4 Miller, T.A. (1970) E. Afr. Med. F. 47, 354-363

5 Nwosu, A.B.C. and Anya, A.O. (1980) Tropenmed. Parasitol. 31, 201-208

6 Goldsmid, J.M. (1965) Centr. Afr. F. Med. 11, 160-167

7 Hoagland, K.E. and Schad, G.A. (1978) Exp. Parasitol. $44,36-49$

8 Hsieh, H.C. (1970) Jap. F. Parasitol. 19, 508-552

9 Knight, R. and Merrett, T.G. (1981) Ann. Trop. Med. Parasitol. 75, 299-314

10 Nawalinski, T. et al. (1978) Am. J. Trop. Med. Hyg. 27, 1152-1161

11 Udonsi, J.K. (1984) Ann. Trop. Med. Parasitol. 78, 443 444

12 Cort, W. W. et al. (1923) Am. F. Hyg. 3, 85-110

13 Schad, G.A. et al. (1984) in Human Ecology and Infectious Diseases (Croll, N.A. and Cross, J, eds), pp. 188223, Academic Press, New York

14 Udonsi, J.K. et al. (1980) Zeitschr. Parasitenk. 63, 251259

15 Maplestone, P.A. (1932) Ind. F. Med. Res. 19, 11451151

16 Nawalinski, T. et al. (1978) Am. J. Trop. Med. Hyg. 27, 1162-1173

17 Smillie, W.G. (1922) Monogr. Rockefeller Inst., Second Paper 17, 1-73
18 Palmer, E.D. (1955) Am. J. Trop. Med. Hyg. 4, 756-757

19 Keymer, A.E. and Slater, A.F.G. (1987) Parasitol. Today 3, 56-58

20 Schad, G.A. et al. (1973) Science 180, 502-504

21 Nawalinski, T.A. and Schad, G.A. (1974) Am. F. Trop. Med. Hyg. 23, 895-898

22 Maplestone, P.A. (1930) Ind. F. Med. Res. 18, 685-698

23 Schad, G.A. and Anderson, R.M. (1985) Science 228, 1537-1540

24 Haswell-Elkins, M.R. et al. Parasitology (in press)

25 Kendrick, J.F. (1934) Am. F. Trop. Med. Hyg. 15, 363379

26 Miller, H.R.P. (1984) Vet. Immunol. Immunopathol. 6, $167-259$

27 Stewart, D.F. (1955) Nature 176, 1273-1274

28 Dineen, J.K. et al. (1977) Int. F. Parasitol. 7, 211-215

29 Wakelin, D. (1985) Parasitol. Today 1, 17-23

30 Stewart, D.F. (1950) Austr. F. Agric. Res. 1, 301-321

31 Behnke, J.M. (1987) in Human Parasitic Diseases. Hookworms. (Gillies, H.M., ed.), Elsevier Science Publishers, Amsterdam (in press)

32 Hill, R.B. (1926) Am. F. Hyg. 6, 103-117

33 Sweet, W.C. (1925) Ceylon F. Sci. Sect. D, Med. Sci. 1, 129-140

34 Seo, B.S. et al. (1980) Korean F. Parasitol. 18, 145-151

35 Kumar, N. et al. (1980) Ind. 7. Med. Res. 71, 531-537

36 Hotez, P. et al. (1985) F. Biol. Chem. 260, 7343-7348

37 Plaut, A.G. (1983) Ann. Rev. Microbiol. 37, 603-622

38 Carroll, S.M. and Grove, D.I. (1985) Exp. Parasitol. 60, 263-269

39 Behnke, J.M. (1987) Adv. Parasitol. (in press)

40 Stokes, C.R. (1984) in Local Immune Responses of the Gut (Newby, T.J. and Stokes, C.R., eds), pp. 97-141, CRC Press, Florida, USA

41 Kalkofen, U.P. (1974) Am. 7. Trop. Med. Hyg. 23. 1046-1053

42 Klaver-Wesseling, J.C.M. et al. (1978) Zeitschr. Parasitenkd. 56, 147-157

43 McLaren, D.J. (1976) Adv. Parasitol. 14, 195-265

44 Behnke, J.M. et al. (1986) Trans. R. Soc. Trop. Med. Hyg. 80, 146-149

45 Schad, G.A. et al. (1975) in Nuclear Techniques in Helminthology Research pp. 41-54, Int. Atomic Energy Agency, Vienna

46 Carr, A. and Pritchard, D.I. (1987) Mol. Biochem. Parasitol. 19, 251-258

\section{Asymptomatic Malaria Infections - Do They Matter?}

\section{B.M. Greenwood}

In non-immune patients, most $P$. falciparum infections result in high parasitaemias and severe symptoms unless treated early ${ }^{1}$. Fortunately however, in areas where malaria is endemic and where most of the population have some immunity, this is not the case and severe, clinical infections are relatively uncommon.

What proportion of new malaria infections causes symptoms in semi-immunes is uncertain. In The Gambia, we have found that children who live in a rural area experience about 1 clinical attack of malaria per year $^{2}$ and a similar incidence has been noted in Liberia ${ }^{3}$. In our survey, children were visited only once a week so that mild attacks may have gone unrecorded between visits. But even in areas of high malaria transmis- sion, it is unlikely that children experience more than 2 clinical attacks of malaria per year. In our study area in The Gambia, children are exposed to about 50 bites from infected mosquitoes per year (S. Lindsay $e t$ al. unpublished) but in other parts of Africa, where transmission is more intense, inoculation rates 2 or 3 times higher have been recorded ${ }^{4}$. Not all sporozoite inoculations lead to infection but these entomological observations suggest that in endemic areas, only a small proportion of malaria infections reach a sufficiently high parasitaemia to cause an acute illness (Fig. 1). The remainder produce a low-level, asymptomatic parasitaemia which may last for weeks or months ${ }^{5}$. Are these asymptomatic infections of any clinical sig- 